\title{
The Correlation Between Nurses' Work Engagement and Workplace Incivility
}

\author{
Leila Hosseinpour-Dalenjan, ${ }^{1}$ Foroozan Atashzadeh-Shoorideh, ${ }^{2,}{ }^{*}$ Meimanat Hosseini, ${ }^{3}$ and Jamileh
}

\author{
Mohtashami ${ }^{4}$ \\ ${ }^{1}$ School of Nursing and Midwifery, Shahid Beheshti University of Medical Sciences, Tehran, IR Iran \\ ${ }^{2}$ Department of Nursing Management, School of Nursing and Midwifery, Shahid Beheshti University of Medical Sciences, Tehran, IR Iran \\ ${ }^{3}$ Department of Community Health Nursing, School of Nursing and Midwifery, Shahid Beheshti University of Medical Sciences, Tehran, IR Iran \\ ${ }^{4}$ Psychiatric Nursing Department, School of Nursing and Midwifery, Shahid Beheshti University of Medical Sciences, Tehran, IR Iran \\ "Corresponding author: Foroozan Atashzadeh-Shoorideh, Department of Nursing Management, School of Nursing and Midwifery, Shahid Beheshti University of Medical \\ Sciences, Tehran, IR Iran. Tel: +98-2188655366, Fax: +98-2188202521, E-mail: f_atashzadeh@sbmu.ac.ir
}

Received 2017 January 05; Revised 2017 January 27; Accepted 2017 February 05.

\begin{abstract}
Background: Because nurses' work engagement is related to positive outcomes like increasing organization productivity, it is necessary to promote it. The first step to achieve this goal is to determine the factors associated with nurses' work engagement, yet very few studies have been conducted on this subject in Iran.

Objectives: This study aimed at investigating the correlation between work engagement and workplace incivility in nurses who were working in selected teaching hospitals of Shahid Beheshti University of Medical Sciences in Tehran, Iran, during 2015 and 2016. Methods: In this descriptive correlational study, 516 nurses were selected randomly from teaching hospitals of Shahid Beheshti University of Medical Sciences during 2015 and 2016. Data were collected using the demographic questionnaire, the utrecht work engagement scale, and the nursing incivility scale.

Results: The means of work engagement and workplace incivility scores were $3.59 \pm 1.16$ (average) and $2.68 \pm 0.65$ (low), respectively. A significant negative correlation was found between work engagement and workplace incivility in nurses $(P<0.01, r=-0.27)$. The findings of the present study revealed that the nurses' work engagement and incivility from physicians were significantly different based on the type of ward. In addition, the mean score of incivility from physicians were significantly different based on different hospitals and work experience.

Conclusions: Nursing managers should use proper strategies to improve nurses' work engagement and decrease the incidence of incivility in hospitals and clinical environments.
\end{abstract}

Keywords: Work Engagement, Workplace Incivility, Nurses

\section{Background}

The organizations are an important part of people's life as they spend many hours of their lives in these places. The quality of spending these relatively long hours is a very important issue because it directly influences the staff morale and indirectly affects the employees' interaction with the families and the community. If the employees of an organization work enthusiastically and be emotionally attached to their job and organization, not only the organization will be more successful in achieving its objectives but also the community will become enthusiastic (1).

Work engagement is an active and positive mental state associated with work (2), and it is useful directly and indirectly for both organizations and the public (3). The benefits for the organizations are as follow: reducing employees' absenteeism; improving the employees' morale; increasing safety; more capability for the use of local volunteers by using succession plans; reducing the em- ployee's turnover; and increasing the employees' motivation (4). Moreover, the benefits outside the organization are as follow: increasing productivity; increasing profit and revenue; increasing customer loyalty; and increasing the ability to attract talented staff from outside the organization. In addition, increasing staff productivity, higher wages, increasing self-esteem, and improving the level of employees' health are the positive effects of work engagement on employees (3).

Adams found that those nurses who have more work engagement, have better performance and are more interested in their job. Moreover, they are more stable when they are faced with difficulties and hardness of work, and this directly affects the success of the health system (5). In Montgomery et al. (6) and Bogaert et al. (7) studies, nurses' work engagement was moderate to high. However, in Iran, Alipour-Birgani (8) and Keyvanara et al. (9) found that the mean scores of nurses' work engagement were medium 
and low, respectively.

Keyvanara et al. (9) showed a significant positive relationship between work engagement and positive organizational behavior among nurses. In addition, Naderi and Safarzade (10) found a significant positive correlation between work engagement and organizational citizenship behavior.

In addition to positive organizational behaviors, there is another type of organizational behavior in the workplace called "negative behaviors". Negative behaviors have a different domain and intensity; some of them have high intensity and the others are subtle and small. However, many theorists argue that most negative behaviors in the workplace occur with low intensity (11). Workplace incivility is a negative behavior with low-intensity and unclear intention that violates the required norms for mutual respect in the workplace. Violation of norms, unclear intention, and low-intensity are 3 key features of workplace incivility (12). Examples of these behaviors include sarcastic talking and mocking, humiliating opinion and tone of voice, hostility, staring at others (13), interrupting someone's conversation, and ignoring the strength and identity of the person in the presence of other associates (14).

Furthermore, incivility is an environmental stressor that can intensify the effects of other environmental stressors (15) and it brings up many problems and complications for the nurses and the patients (16). Simons (17) found a significant relationship between nurses' workplace incivility and the desire to leave the organization. Wilson et al. (18) concluded that the nurses who are experienced or observed the negative behaviors were thinking more about leaving their job. Another study specified that unhealthy work environments lead to increased rates of burnout, medication errors, ineffective care of patients, and high levels of stress in the work environment (19).

Workplace incivility, in addition to the infliction of personal costs, has harmful effects on organization's health and performance. Increasing the measures of nurses' absenteeism, decreasing productivity, and rising the costs associated with them are all the negative organizational outcomes of nurses' workplace incivility (20). Other studies also showed the reduction of productivity $(21,22)$.

Moreover, studies have shown that incivility behaviors in the workplace are common (12). For example, Cortina and Magley (23) found that $75 \%$ of university employees have experienced some form of incivility. Researchers have found that incivility is more common among nurses than other employees (24). Winstanley and Whittington (25) estimated that 9 out of 10 nurses have experienced verbal abuse, a form of incivility behavior, in clinical environments. In addition, in a randomized study conducted by Ulrich et al. in the United States, it was revealed that more than a quarter of nurses had experienced violence (19).

Taylor (26) found a negative correlation between work engagement and incivility among students and graduates of business administration. However, Vagharseyyedin and Salmani-Mud (27) did not find any significant correlation between nurses' work engagement and workplace incivility. Therefore, due to inconsistencies in the studies about the correlation between work engagement and workplace incivility, it was deemed necessary to examine the relationship between these 2 variables.

\section{Objectives}

This study aimed at determining the correlation between work engagement and workplace incivility in nurses working in teaching hospitals of Shahid Beheshti University of Medical Sciences in Tehran, Iran, during 2015 and 2016.

\section{Materials and Methods}

\subsection{Design, Setting and Participants}

In this descriptive correlational study with the assumption of correlation between work engagement and workplace incivility based on Taylor's (26) study and $\mathrm{r}=$ 0.15 , a sample size of 469 participants was obtained using the following formula with a predicted subject attrition rate of $10 \%$. Thus, the total number of samples required was 516. A total of 550 questionnaires were distributed and 516 nurses completed the questionnaires (93.8\% response rate).

First, 8 hospitals were selected randomly using the random numbers table. These hospitals were governmental hospitals and not referral centers. In this study, 516 nurses working in teaching hospitals of Shahid Beheshti University of Medical Sciences in Tehran were selected randomly. The inclusion criteria were as follow: holding at least a BSN degree in nursing, having at least 6 months of clinical work experience, and providing informed written consent. The exclusion criterion was defects in the completed questionnaires.

\subsection{Ethical Considerations and Data Collection}

After receiving the necessary approvals from the university and research environments and the ethics code of SBMU2.REC.1394.163 from the committee of ethics in research at Shahid Beheshti University of Medical Sciences, the researcher attended the research environments in 3 shifts of morning, evening, and night. After explaining the study objectives and emphasizing the confidentiality 
of the information to the participants, the researchers obtained informed consents for participation in the research. The demographic questionnaire included variables such as age, sex, marital status, education level, type of employment, type of ward, type of work shift, and work experience. In addition, the Utrecht Work Engagement Scale and the Nursing Incivility Scale were distributed randomly to the eligible nurses. Enough time was given to the participants to complete the questionnaires. The questionnaires were collected at the next visit determined by the participants. The sampling process began in December 2015 and ended in March 2016 in Tehran.

\subsection{Measurements}

The Utrecht work engagement scale was designed by Salanova and Schaufeli in 2001 at the University of Utrecht Netherlands with 17 items in 3 dimensions of vigor (Items 1 to 6 ), dedication (Items 7 to 11), and absorption (Items 12 to 17). Each item is rated on a seven-point Likert scale ranging from never (0), almost never (1), rarely ( 2), sometimes (3), often (4), very often (5), and always (6). The mean scores for each dimension and the mean of the total score are reported between 0 to 6 . Higher scores indicate the higher levels of work engagement in participants (28). According to Schaufeli and Bakker's (28) study, the total score of work engagement of 1.93 and less is rendered as very low engagement, the total score of work engagement of 1.94 - 3.06 as low engagement, the total score of work engagement of 3.07 - 4.66 as average engagement, the total score of work engagement of 4.67 - 5.53 as high engagement, and total score up to 5.54 as very high engagement.

The validity of this tool with high validity and reliability has been confirmed in a research conducted by Taghipour (29) in Iran. Cronbach's alpha coefficients for the scale and each of the dimensions of vigor, dedication, and absorption were $0.94,0.86,0.88$ and 0.86 , respectively. In the present study, Cronbach's alpha coefficients for the scale and for each of the dimensions of vigor, dedication, and absorption were $0.94,0.84,0.91$ and 0.86 , respectively.

The nursing incivility scale was designed by Guidroz et al. (30) with the participation of 163 nurses working in the United States of America with 43 items including sources of incivility which are as follow: general incivility ( 9 items), nurse incivility (10 items), supervisor incivility (7 items), physician incivility (7 items), and patient/visitor incivility (10 items). This scale assesses the hospital nurses' experiences with incivility. Each item is rated on a five-point Likert scale ranging from strongly disagree (1) to strongly agree (5), and the mean score of each source is reported (30).

In Iran, Kalantari et al. (31) validated this scale and reduced its items to 37: general incivility (8 items), nurse in- civility (9 items), supervisor incivility (5 items), physician incivility ( 6 items), and patient/visitor incivility (9 items). Cronbach's alpha coefficient of the scale was 0.86. In the present study, the Persian version of the Nursing Incivility Scale with 37 items was used, and exploratory and confirmatory factor analyses were conducted to determine its validity. Cronbach's alpha coefficient of the scale and of each source of general incivility, nurse incivility, supervisor incivility, physician incivility, and patient/visitor incivility was $0.95,0.86,0.93,0.92,0.87$ and 0.85 , respectively.

\subsection{Data Analysis}

Data were analyzed using SPSS 20. The descriptive statistics (mean, standard deviation and relative frequency) were used to determine and evaluate the features of each demographic information and calculate the mean score of the study variables. Considering the nonnormal distribution of the data based on KolmogorovSmirnov test, the Spearman correlation coefficient was used to determine the correlation between nurses' work engagement and workplace incivility in this research. The Kruskal-Wallis and U-Mann-Whitney tests were used to analyze and determine the correlation between work engagement and incivility with demographic data $(\mathrm{P}<0.05)$.

\section{Results}

Of the total participants, $81.8 \%$ were female, $55.2 \%$ were married, and 90.3\% held a bachelor's degree in nursing. Nurses' age ranged from 21 to 60 years with a mean (SD) of $32.35 \pm 7.44$. Their work experience ranged from 1 to 30 years with a mean (SD) of $8.65 \pm 6.96$. Most participants (45.3\%) were official employees, (65.3\%) in circulating shifts, (25.6\%) in the surgery ward, and (27.9\%) in the hospital $\mathrm{H}$.

In this study, the overall mean scores of nurses' work engagement and workplace incivility were $3.59 \pm 1.16$ and $2.68 \pm 0.65$, respectively, representing work engagement and workplace incivility experience among the participants, which were average and low, respectively. A negative correlation was detected between work engagement and workplace incivility of the participants in the present study $(\mathrm{P}<0.01, \mathrm{r}=-0.27)$ (Table 1$)$.

The highest work engagement mean score was in ICUs and the lowest in NICUs, with a statistically significant difference $(P=0.01)$ (Table 2). Among the different sources of incivility, the physician incivility mean score (3.09) was greater than others (Table 1 ). Moreover, the physician incivility mean score (3.44) was greater among the nurses with 26-30 years of work experience, which was statistically significant $(\mathrm{P}=0.04)$ (Table 3$)$. The physician incivility mean score was mostly (4.09) in the NICUs and it was the least 
Table 1. Work Engagement and Workplace Incivility Mean of Scores and Spearman Correlational Coefficients Between Variables

\begin{tabular}{|c|c|c|c|c|c|c|c|c|c|c|c|c|c|c|}
\hline & Variables & Mean \pm SD & Median & IQR & 1 & 2 & 3 & 4 & 5 & 6 & 7 & 8 & 9 & 10 \\
\hline 1 & Work engagement & $3.59 \pm 1.16$ & 3.62 & 1.54 & - & & & & & & & & & \\
\hline 2 & Vigor & $3.46 \pm 1.20$ & 3.50 & 1.47 & $0.86^{\mathrm{a}}$ & - & & & & & & & & \\
\hline 4 & Absorption & $3.50 \pm 1.27$ & 3.50 & 1.67 & $0.89^{\mathrm{a}}$ & $0.68^{\mathrm{a}}$ & $0.73^{\mathrm{a}}$ & - & & & & & & \\
\hline 5 & Nursing incivility scale & $2.68 \pm 0.65$ & 2.72 & 0.82 & $-0.27^{\mathrm{a}}$ & $-0.21^{\mathrm{a}}$ & $-0.24^{\mathrm{a}}$ & $-0.24^{\mathrm{a}}$ & - & & & & & \\
\hline 6 & General incivility & $2.51 \pm 0.78$ & 2.50 & 1.13 & $.010^{\mathrm{b}}$ & -0.05 & $-0.12^{\mathrm{a}}$ & -0.07 & $0.64^{\mathrm{a}}$ & - & & & & \\
\hline 7 & Nurse incivility & $2.41 \pm 0.89$ & 2.33 & 1.11 & $-0.11^{b}$ & -0.06 & $-0.10^{\mathrm{b}}$ & $-0.12^{\mathrm{a}}$ & $0.72^{\mathrm{a}}$ & $0.62^{\mathrm{a}}$ & - & & & \\
\hline 8 & Supervisor incivility & $2.46 \pm 0.98$ & 2.40 & 1.00 & $-0.26^{\mathrm{a}}$ & $-0.24^{\mathrm{a}}$ & $-0.22^{\mathrm{a}}$ & $-0.23^{\mathrm{a}}$ & $0.74^{\mathrm{a}}$ & $0.39^{\mathrm{a}}$ & $0.48^{\mathrm{a}}$ & - & & \\
\hline 9 & Physician incivility & $3.09 \pm 1.0$ & 3.17 & 1.50 & $-0.20^{\mathrm{a}}$ & $-0.17^{\mathrm{a}}$ & $-0.17^{\mathrm{a}}$ & $-0.17^{\mathrm{a}}$ & $0.68^{\mathrm{a}}$ & $0.19^{\mathrm{a}}$ & $0.26^{\mathrm{a}}$ & $0.35^{\mathrm{a}}$ & - & \\
\hline 10 & Patient/visitor incivility & $2.91 \pm 0.90$ & 3.00 & 1.22 & $-0.33^{\mathrm{a}}$ & $-0.24^{\mathrm{a}}$ & $-0.30^{\mathrm{a}}$ & $-0.32^{\mathrm{a}}$ & $0.70^{\mathrm{a}}$ & $0.24^{\mathrm{a}}$ & $0.30^{\mathrm{a}}$ & $0.37^{\mathrm{a}}$ & $0.57^{\mathrm{a}}$ & . \\
\hline
\end{tabular}

(2.31) in the psychiatric ward, which was statistically significant $(\mathrm{P}=0.01)$ (Table 4$)$. The difference between the physician incivility mean scores in different hospitals was statistically significant $(\mathrm{P}=0.007)$ so that the nurses working in hospital C (3.47) had the highest and those in hospital $\mathrm{G}$ and hospital A (2.87) had the least incivility from physicians (Table 5).

\section{Discussion}

The findings of the present study revealed that the mean score for overall work engagement was 3.59, while it was $3.46,3.82$, and 3.50 for each of the dimensions of vigor, dedication, and absorption, respectively. According to Schaufeli and Bakker's (28) classification, nurses participating in this study had an average level of work engagement.

Consistent with the findings of the current study, other researchers such as Vagharseyyedin and Salmani-Mud (27), Akharbin et al. (5), and Alipour-Birgani (8) found that the overall mean score of work engagement to be at the moderate level. Nonetheless, in other studies such as White et al. (32), Bogaert et al. (7), and Montgomery et al. (6), the overall mean score of nurses' work engagement was higher than the value reported in the present study. Furthermore, Lu et al. (33) reported a value lower than this study.

To justify the inconsistencies with the results of the above studies, we should consider the role of job resources in the staff work engagement. Several studies have shown a positive correlation between job resources and work engagement (2). Job resources including independence, social support from colleagues, diversity of skills (34), performance feedback, learning opportunities (35), and innovation (36) refer to all physical, social, and organizational aspects of work, which may reduce job demands and other associated physical and psychological costs. Moreover, all of them may be effective in achieving the goals and stimulating personal growth, learning, and development (37).

Our findings showed that the overall mean score of incivility was 2.68 in the present study. In Hutton and Gates' (38) study, the overall mean score for incivility in direct care staff was 2.13 out of 5 , which is consistent with the present study. In Kalantari et al. (31) study, the overall mean score for incivility among nurses was not (3.89) consistent with this study.

This mismatch between the current study and Kalantari et al. (31) study will be clarified by investigating the factors affecting the workplace incivility. Humans are complex creatures with different experiences of family and work life such as education, cultural, ethnic, racial, and gender prejudice. Thus, understanding the hierarchy and roles, personal values, communication styles, personality disorders, and other events affect the mood, attitude, and practice, all of which influence the destructive behaviors at work (39). Stress, high workload, lack of job security, organizational change, poorly-organized work, social power differences, interdependence of tasks (40), competition (41), and irregularities of organization (42) are the factors affecting the workplace incivility.

In this study, the mean score of physician incivility was higher than the other sources of incivility. In Kalantari et al. (31) study, the highest experienced incivility was interaction with physicians (4.38 out of 5 ), which is consistent with the findings of the present study. Therefore, it is necessary to pay more attention to behavioral interactions between physicians and nurses.

Patient/visitor incivility ranked second with the mean score of 2.91. Kalantari et al. (31) reported the mean score 3.85 out of 5 that is greater than this study, but it ranked fourth among the other sources of incivility. Guidroz et al. (30) and Smokler Lewis (20) reported the mean scores for patient/visitor incivility as 2.25 and 2.39 , respectively, 
Table 2. Work Engagement and Type of Ward

\begin{tabular}{llll}
\hline & & & Work Engagement \\
Ward & Mean \pm SD & PValue & df \\
\hline Medical & $3.68 \pm 1.10$ & & Kruskal-Wallis \\
Surgical & $3.53 \pm 1.21$ & & 7.0 \\
\hline CCU & $3.51 \pm 1.03$ & 0.01 & 17.50 \\
ICU & $3.76 \pm 1.20$ & & \\
\hline ED & $3.63 \pm 1.05$ & & \\
\hline NICU & $2.68 \pm 0.87$ & & \\
\hline Pediatrics & $2.80 \pm 1.43$ & & \\
\hline Psychiatry & $3.53 \pm 1.37$ & & \\
\hline
\end{tabular}

Abbreviations: CCU, cardiac care unit; ED, emergency department; ICU, intensive care unit; NICU, neonatal intensive care unit.

Table 3. Physician Incivility and Work Experience

\begin{tabular}{|c|c|c|c|c|}
\hline \multirow[b]{2}{*}{ Work Experience, $y$} & \multicolumn{4}{|c|}{ Physician Incivility } \\
\hline & Mean \pm SD & PValue & df & Kruskal-Wallis \\
\hline $1-5$ & $3.14 \pm 0.96$ & \multirow{5}{*}{0.04} & \multirow{5}{*}{5.0} & \multirow{5}{*}{11.48} \\
\hline $6-10$ & $3.03 \pm 1.09$ & & & \\
\hline 11- 15 & $3.14 \pm 0.99$ & & & \\
\hline $21-25$ & $2.54 \pm 0.89$ & & & \\
\hline $26-30$ & $3.44 \pm 0.62$ & & & \\
\hline
\end{tabular}

Table 4. Physician Incivility and Type of Ward

\begin{tabular}{|c|c|c|c|c|}
\hline \multirow[t]{2}{*}{ Ward } & \multicolumn{4}{|c|}{ Physician Incivility } \\
\hline & Mean \pm SD & PValue & df & Kruskal-Wallis \\
\hline Medical & $3.00 \pm 0.98$ & \multirow{8}{*}{0.01} & \multirow{8}{*}{7.0} & \multirow{8}{*}{17.79} \\
\hline Surgical & $3.05 \pm 0.90$ & & & \\
\hline CCU & $3.12 \pm 0.97$ & & & \\
\hline ICU & $3.18 \pm 0.97$ & & & \\
\hline ED & $3.11 \pm 1.10$ & & & \\
\hline NICU & $4.09 \pm 0.97$ & & & \\
\hline Pediatrics & $3.11 \pm 1.15$ & & & \\
\hline Psychiatry & $2.31 \pm 1.09$ & & & \\
\hline
\end{tabular}

which were lower than the mean score in this study, however, it ranked fourth among the other sources of incivility. Norris (43) believes that when patients and their families are unstable in the emotional and psychological aspects, they may show violent behaviors against the nurses.

In this study, the lowest amounts of incivility belonged to nurse incivility and supervisor incivility with the mean scores of 2.41 and 2.46, respectively. In Kalantari et al. (31), the lowest amount of incivility belonged to supervisor incivility with the mean score of 2.98 out of 5. In Guidroz et al. (30) and Smokler Lewis (20) studies, the mean scores of supervisor incivility were 1.84 and 2.18 , respectively. Generally, studies show low levels of incivility behaviors from nursing managers, which may be attributed to their power 
Table 5. Physician Incivility and Hospital

\begin{tabular}{llll}
\hline Hospital & & Physician Incivility & df \\
& Mean \pm SD & PValue & \\
\hline A & $2.87 \pm 0.95$ & & \\
\hline B & $2.93 \pm 0.96$ & & 7.0 \\
\hline C & $3.47 \pm 1.05$ & 0.007 & \\
\hline D & $3.19 \pm 1.08$ & & 19.60 \\
\hline E & $2.97 \pm 1.03$ & & \\
\hline F & $3.02 \pm 1.09$ & & \\
\hline G & $2.87 \pm 0.94$ & & \\
H & $3.27 \pm 0.91$ & \\
\hline
\end{tabular}

and responsibility against other nurses. The nursing managers are obliged to observe the rules and regulations of the organization and should prevent the occurrence of such behaviors, and if such actions occurred, they should adopt coping strategies.

Additionally, the results revealed a negative correlation between work engagement and workplace incivility among nurses participating in this study. Taylor(26) found a significant negative correlation between workplace incivility and work engagement $(\mathrm{P}<0.05, \mathrm{r}=-0.15)$, which was consistent with our findings. However, Vagharseyyedin and Salmani-Mud (27) found no significant correlation between workplace incivility and work engagement $(\mathrm{P}>$ 0.05) that was not in line with our findings. To justify the findings of the study above, we may add that perhaps some factors like independence, thankfulness, and creative climate affected the work engagement of the nurses participating in this study. In Vagharseyyedin and Salmani-Mud (27) study, one of the nurses said, "We are faced with many different problems in the ward although such behaviors by a colleague or supervisor are unimportant."

However, this cannot reduce the importance of nurses' workplace incivility. Generally, creating and maintaining a safe and healthy workplace is a part of the responsibilities of the nurses. However, having sufficient knowledge about the harmful effects of these behaviors and knowing how to stop it can enable the nurses to create a respectful, healthy, and safe workplace and protect the patients from complications of negative behaviors (44).

The highest amount of work engagement belonged to ICUs and the lowest to NICUs, with a statistically significant difference. However, Vagharseyyedin and Salmani-Mud (27) did not find any significant differences in the work engagement of those nurses working in a different ward, which was not consistent with our findings. The findings revealed that job resources such as workmates interaction, creative working environment, and appropriate feedback are beneficial in promoting the work engagement even under conditions of high job demands such as high workload and poor physical environment $(2,34)$. These findings justify a significant difference in the mean score of work engagement in nurses in different wards.

The results also revealed that the mean score of incivility manifested by physicians was significantly different between the nurses working in different wards, thus, incivility behaviors by physicians in NICUs were the most and in the psychiatry wards were the least. Regardless of the differences in the type of wards, this is consistent with findings of Smokler Lewis (20). Perhaps, this is because of the greater sensitivity of treatment and care issues in NICUs than other wards and the importance of care in these wards that drives the physicians to exhibit more stressful and incivility behaviors.

The mean score of physician incivility from the viewpoint of the nurses working in various hospitals was also significantly different. This means that those nurses working in hospital $\mathrm{C}$ reported the most incivility and those in hospital G and hospital A reported the least incivility on the part of physicians. This may be attributed to a number of organizational factors such as competition in the organization (41), organizational change (40), irregularities in the organization (42), and organizational culture that are effective in the workplace incivility.

Considering the work experience of the nurses in the present study, the mean score of physician incivility from the viewpoint of nurses with 26 - 30 years of work experience was greater than all, which was statistically significant and inconsistent with the findings of Smokler Lewis (20). Our findings may be attributed to the point that as the nurses' work experience increases, they are more apt to the risk of verbal and nonverbal abuses.

Incivility can affect the nurses and quality of the nurs- 
ing care. The levels of productivity of the organization may be increased if incivility is diminished in hospitals. This study is unique compared to previous studies on incivility in hospitals because for the first time it showed a significant relationship between work engagement and workplace incivility in Iranian hospitals. This study contributes to an understanding of the role of work engagement in creating a positive work environment and work-life balance. It is hoped that by considering the results of this research, the Ministry of Health, Treatment, and Medical Education try to create suitable conditions to increase work engagement and reduce incivility at clinical environments.

\subsection{Limitations}

The present study had several limitations. First, given that the study population was only selected from one city, we could not generalize the results to the entire nursing community of Iran. Thus, it is suggested that future researches be conducted selecting samples from different cities of the country. Second, due to the nature of the descriptive correlational design of this study, correlations between the variables did not indicate a causal relationship. Therefore, it is recommended that future studies use a longitudinal design to investigate the causal relationship between the variables.

\subsection{Conclusions}

We believe that nurses' work engagement can be created and maintained with a moral and free of incivility behavior work environment. Therefore, continuous monitoring of clinical environment in the incidence of incivility behaviors and trying to solve the causative agents would be effective in improving nurses' work engagement, which in turn can improve the effectiveness of services.

\section{Acknowledgments}

This article was the result of postgraduate thesis, which was conducted under the supervision of Dr. Foroozan Atashzadeh-Shoorideh in Shahid Beheshti University of Medical Sciences. This study was a research proposal that had been approved by Shahid Beheshti University of Medical Sciences. The authors would like to express their appreciation to the participants for their valuable contribution.

\section{Footnotes}

Authors' Contribution: Data collection: Leila Hosseinpour-Dalenjan; biostatistics analysis and study design: Leila Hosseinpour-Dalenjan and Foroozan
Atashzadeh-Shoorideh; final revision and editing the manuscript: Meimanat Hosseini and Jamileh Mohtashami.

Conflict of Interest: The authors declare that there is no conflict of interest.

\section{References}

1. Mirzadarani H. Work Engagement window into the social vitality. Soc Sci J. 2013;62:62-70.

2. Bakker AB. An Evidence-Based Model of Work Engagement. Curr Direct Psychol Sci. 2011;20(4):265-9. doi: 10.1177/0963721411414534.

3. Gibbons JM. Employee engagement: A review of current research and its implications. New York: Conference Board; 2006.

4. Askari M. Determining The Impact of Talent Management Strategy on Emotional-Mental Involvement of Employees of Isfahan Municipality. Isfahan: Isfahan University; 2011.

5. Akharbin P, Zahed Babolan A, Naghizadeh Baghi A. The Relationship between Servant Leadership \& Organizational Learning and Nurses' Work Engagement. J Res Dev Nurs Midwifery. 2014;11(1):91-8.

6. Montgomery A, Spanu F, Baban A, Panagopoulou E. Job demands, burnout, and engagement among nurses: A multi-level analysis of ORCAB data investigating the moderating effect of teamwork. Burn Res. 2015;2(2-3):71-9. doi: 10.1016/j.burn.2015.06.001. [PubMed: 26877971].

7. Van Bogaert P, van Heusden D, Timmermans O, Franck E. Nurse work engagement impacts job outcome and nurse-assessed quality of care: model testing with nurse practice environment and nurse work characteristics as predictors. Front Psychol. 2014;5:1261. doi: 10.3389/fpsyg.2014.01261. [PubMed: 25431563].

8. Allipour Birgani S. The Relationship of Work Engagement and Work Stress with Satisfaction from Daily Lives of Nurses in Ahvaz Governmental Hospitals. Jentashapir J Health Res. 2013;4(2):141-9.

9. Keyvanar M, Shahpouri S, Oreyzi HR. Relationship among Organizational Justice, Work Engagement and Positive Organizational Behavior of Nurses via Mediation of their Personal Career Goals. Iran J Nurs. 2014;27(88):22-33.

10. Naderi F, Safarzade S. The Relationship of Organizational Justice, Organizational Health, Job Engagement and Organizational Climate with Psychological Empowerment and Organizational Citizenship Behavior. Knowledge Res Appl Psychol. 2014;15(2):56-68.

11. Stewart SM, Bing MN, Davison HK, Woehr DJ, McIntyre MD. In the eyes of the beholder: A non-self-report measure of workplace deviance. J Appl Psychol. 2009;94(1):207-15. doi: 10.1037/a0012605. [PubMed: 19186905].

12. Arab N, Sheykhshabani EH, Beshlideh K. Antecedents of Workplace Incivility: Investigating some Personal and Organizational Variables $\square$. J Psychol. 2013;17(3):294-309.

13. Lim S, Cortina LM, Magley VJ. Personal and workgroup incivility: impact on work and health outcomes. J Appl Psychol. 2008;93(1):95-107. doi: 10.1037/0021-9010.93.1.95. [PubMed:18211138].

14. Kelley S. Dishonorable treatment: Workplace incivility, cultures of honor and work outcomes. United States of America: Western Kentucky University; 2007.

15. Montalvo L. Civility and Incivility between Nurses and Administrators in VA: A Model to Drive Successful Health Care Outcomes in the Department of Veterans Affairs. United States of America: University of Maryland; 2012.

16. Pearson CM. Research on workplace incivility and its connection to practice. ; 2010.

17. Simons S. Workplace bullying experienced by Massachusetts registered nurses and the relationship to intention to leave the organization. ANS Adv Nurs Sci. 2008;31(2):E48-59. doi: 10.1097/01.ANS.0000319571.37373.d7. [PubMed: 18497581]. 
18. Wilson BL, Diedrich A, Phelps CL, Choi M. Bullies at work: the impact of horizontal hostility in the hospital setting and intent to leave. J Nurs Adm. 2011;41(11):453-8. doi: 10.1097/NNA.0b013e3182346e9o. [PubMed: 22033314].

19. Wu M. Perceptions of Work Engagement of Nurses in Taiwan. United States of America: University of Texas; 2010.

20. Smokler Lewis P. Individual and organizational factors that predict workplace incivility: Impact on costs, absenteeism and productivity. United States of America: Texas Woman's University; 2009.

21. Smokler Lewis P, Malecha A. The impact of workplace incivility on the work environment, manager skill, and productivity. J Nurs Adm. 2011;41(7-8 Suppl):S17-23. doi: 10.1097/NNA.0b013e318221c2a8. [PubMed: 21799351].

22. Berry PA, Gillespie GL, Gates D, Schafer J. Novice nurse productivity following workplace bullying. J Nurs Scholarsh. 2012;44(1):80-7. doi: 10.1111/j.1547-5069.2011.01436.x. [PubMed: 22339938].

23. Cortina LM, Magley VJ. Patterns and profiles of response to incivility in the workplace. J Occup Health Psychol. 2009;14(3):272-88. doi: 10.1037/a0014934. [PubMed: 19586222].

24. Rosenstein AH, O'Daniel M. Disruptive behavior and clinical outcomes: perceptions of nurses and physicians. Am J Nurs. 2005;105(1):54-64. [PubMed: 15659998] quiz 64-5.

25. Winstanley S, Whittington R. Anxiety, burnout and coping styles in general hospital staff exposed to workplace aggression: a cyclical model of burnout and vulnerability to aggression. Work Stress. 2002;16(4):302-15.

26. Taylor SG. Cold looks and hot tempers: Individual-Level Effects of Incivility in the Workplace. United States of America: Bradley University; 2010.

27. Vagharseyyedin SA, Salmani-Mud M. Relationship between nurses' work engagement, structural empowerment, and workplace incivility. Q J Nurs Manag. 2015;4(1):18-27.

28. Schaufeli WB, Bakker AB. Utrecht work engagement scale: Preliminary manual. Utrecht: Occupational Health Psychology Unit, Utrecht University; 2003.

29. Taghipour A. The relationship between organizational culture with work motivation, job engagement and innovative behaviors by mediating of psychological empowerment. Ahvaz: University of Shahid Chamran; 2009.

30. Guidroz AM, Burnfield-Geimer JL, Clark O, Schwetschenau HM, Jex SM. The nursing incivility scale: development and validation of an occupation-specific measure. J Nurs Meas. 2010;18(3):176-200. [PubMed: 21290924].
31. Kalantari S, Hekmatafshar M, Jouybari LM, Sanagoo A, Mohammadi $\mathrm{R}$. Workplace behaviors and its correlation with demographic characteristics: Perspective of nurses in teaching hospitals in Gorgan. $J$ Health Promot Manag. 2012;1(4):7-15.

32. White M, Wells JS, Butterworth T. The impact of a large-scale quality improvement programme on work engagement: preliminary results from a national cross-sectional-survey of the 'Productive Ward'. Int J Nurs Stud. 2014;51(12):1634-43. doi: 10.1016/j.ijnurstu.2014.05.002. [PubMed: 24890896].

33. Lu CQ, Siu OL, Chen WQ, Wang HJ. Family mastery enhances work en gagement in Chinese nurses: A cross-lagged analysis. J Vocation Behav. 2011;78(1):100-9. doi: 10.1016/j.jvb.2010.07.005.

34. Bakker AB, Albrecht SL, Leiter MP. Key questions regarding work engagement. Eur J Work Organiz Psychol. 2011;20(1):4-28. doi: 10.1080/1359432x.2010.485352.

35. Albrech SL. Handbook of employee engagement: Perspectives, issues, research and practice. Human Resource Manag Int Digest. 2011;19(7)

36. Hakanen JJ, Bakker AB, Demerouti E. How dentists cope with their job demands and stay engaged: the moderating role of job resources. EurJOral Sci. 2005;113(6):479-87. doi:10.1111/j.1600-0722.2005.00250.x. [PubMed: 16324137].

37. Bakker AB, Demerouti E. The Job Demands-Resources model state of the art. J Manag Psychol. 2007;22(3):309-28. doi: $10.1108 / 02683940710733115$

38. Hutton S, Gates D. Workplace incivility and productivity losses among direct care staff. AAOHN J. 2008;56(4):168-75. [PubMed 18444405].

39. Rosenstein AH, O'Daniel M. Impact and implications of disruptive behavior in the perioperative arena. J Am Coll Surg. 2006;203(1):96-105. doi: 10.1016/j.jamcollsurg.2006.03.027. [PubMed: 16798492].

40. Hershcovis MS, Barling J.16 Towards a relational model of workplace aggression. United Kingdom: Edward Elgar; 2007.

41. Alavosius MP. Comments on "There's a Policy for That": Interdisciplinary Analyses of Hostile Work Environments. Behav Soc Issues. 2009;17(2):182. doi:10.5210/bsi.v17i2.2348.

42. Roscigno VJ, Hodson R, Lopez SH. Workplace incivilities: the role of interest conflicts, social closure and organizational chaos. Work Employment Soci. 2009;23(4):747-73. doi: 10.1177/0950017009344875.

43. Norris TL. Lateral violence: is nursing at risk?.Tenn Nurse. 2010;73(2):1. [PubMed: 20608367] 7.

44. McNamara SA. Incivility in nursing: unsafe nurse, unsafe patients. AORN J. 2012;95(4):535-40. doi: 10.1016/j.aorn.2012.01.020. [PubMed: 22464626]. 